\title{
The Preparation and Partial Characterization of Antigenic Fractions Obtained from the Mycelial Walls of Several Aspergillus Species
}

\author{
By VERONICA M. HEARN,* A. G. PROCTOR AND \\ D. W. R. MACKENZIE \\ Mycological Reference Laboratory, London School of Hygiene and Tropical Medicine, \\ Keppel Street, London WC1E $7 H T$
}

(Received 23 November 1979)

\begin{abstract}
Extracts with immunological activity were prepared from Aspergillus fumigatus, $A$. flavus, $A$. terreus, $A$. niger and $A$. nidulans. In each case crude mycelial wall was extracted with an aqueous solution of Triton X-100 giving detergent-soluble material. Further fractionation was achieved by removing the detergent from this solution; the resultant precipitate was removed by centrifugation, and the aqueous supernatant was used as a source of soluble antigens. The sensitivity of these preparations was compared with that of water-soluble antigenic material, prepared from whole macerated mycelium, by double diffusion and counterimmunoelectrophoresis using homologous antisera and sera from patients suffering from aspergilloma and allergic bronchopulmonary aspergillosis. The selectivity of these antigenic preparations was monitored with heterologous antisera raised in rabbits. Batch variability was analysed for one strain of $A$. fumigatus using chemical and immunological methods. The nature of the antigenic sites involved in these reactions was investigated by studying the susceptibility of the preparations to proteolytic hydrolysis, periodate oxidation and concanavalin A treatment. The total protein and carbohydrate content of each fraction was determined and the constituent sugars analysed in an attempt to correlate chemical composition with antigenic activity.
\end{abstract}

\section{INTRODUCTION}

Serological tests are used extensively in the detection of all forms of human aspergillosis (Biguet et al., 1964; Coleman \& Kaufman, 1972; Longbottom \& Pepys, 1964). The antigens which have been used as diagnostic aids have been prepared by different methods and in general they are highly complex mixtures which are difficult to analyse and, hence, to reproduce. With test sera these antigens can give conflicting results due to batch-to-batch variation, lack of sensitivity and cross-reactivity with other antibody species. We have recently described the preparation of antigens from $A$. fumigatus mycelial wall and their reactivity against test sera (Hearn \& Mackenzie, 1979, 1980). The evidence, based on serological and chemical data, indicated an increased sensitivity of these preparations over that of their water-soluble counterpart, used as a routine antigen in our laboratory. A similar fractionation procedure has now been applied to several other species of Aspergillus. The antigenic fractions thus obtained have been studied chemically and serologically with special reference to sensitivity and specificity. 


\section{METHODS}

Preparation of antigens. The following species of Aspergillus were used: A. flavus (NCPF 2008), A. niger (NCPF 2022), A. nidulans (NCPF 2020), A. terreus (NCPF 2026) and two strains of A. fumigatus (NCPF 2109 and 2140). All were grown in neutral glucose peptone medium and the organisms were incubated for $3 \mathrm{~d}$ as described by Proctor (1976). The procedure for preparation and detergent-extraction of crude wall fractions was as reported for $A$. fumigatus (Hearn \& Mackenzie, 1979). In each case this yielded a Tritonsoluble fraction (TE) of wall material. On removal of Triton, a precipitate formed: after centrifugation, the supernatant contained antigenically active material designated as WS/TE (Hearn \& Mackenzie, 1980). Water-soluble antigens (WS) from macerated mycelium were prepared according to Proctor (1976).

Production of antisera. Antiserum was obtained by the hyper-immunization of rabbits with the watersoluble fraction of each $3 \mathrm{~d}$ mycelium. This fraction (at $60 \mathrm{mg} \mathrm{ml}^{-1}$ ) was mixed with an equal volume of Freund's incomplete adjuvant and $1 \mathrm{ml}$ was inoculated subcutaneously at weekly intervals for 2 or 3 weeks. An antiserum raised in a sheep to A. fumigatus strain 2109 was also used.

Serum specimens. Sera were obtained from patients suffering from allergic bronchopulmonary aspergillosis (ABPA) (10 in number) or aspergilloma ( 7 samples). Some were specimens received by our laboratory while the remainder were kindly donated by Dr John Edwards of Sully Hospital in Wales.

Concentration of patients' sera. This procedure was carried out on selected ABPA sera. The bulk of the plasma proteins were precipitated with caprylic acid (Steinbuch \& Audran, 1969). The IgG component (and a portion of the IgA) which remained in the supernatant were concentrated fivefold.:

Double diffusion. Tests were done using $1 \%(\mathrm{w} / \mathrm{v})$ purified agar (Oxoid) in borate buffer at $\mathrm{pH} 8.2$ containing $0.5 \%(\mathrm{w} / \mathrm{v})$ EDTA (Hearn \& Mackenzie, 1979).

Counterimmunoelectrophoresis. In a $2.5 \mathrm{~mm}$ diam. antigen well $5 \mu 1$ of fraction was applied, with $15 \mu 1$ of either a homologous or selected patient's antiserum in a $4.5 \mathrm{~mm}$ diam. well (Mackenzie et al., 1980). After application of a constant current to the gel for approximately $1.5 \mathrm{~h}$, the slides were washed, fixed and stained with Coomassie Brilliant Blue R, C.I. no. 42660.

Chemical analyses. Total neutral reducing sugars were estimated according to Dubois et al. (1956). The procedure of Lee \& Montgomery (1961) was followed for the analysis of hexosamines as neutral sugars after prior deamination. Protein was measured with the Folin Ciocalteau reagent with bovine serum albumin as a standard. Glucose was measured by the hexokinase/glucose-6-phosphate dehydrogenase system (Sigma kit); galactose was estimated using the galactose dehydrogenase method (Boehringer kit).

Gas-liquid chromatography (g.l.c.). Detection of individual monosaccharides present in TE preparations was carried out by analytical g.l.c. kindly performed by Mr R. A. Faulkes (National Institute for Medical Research, Mill Hill, London), according to the method of Clamp (1974).

Hexosamine analysis. Individual hexosamines were determined on an amino acid analyser. The analyses were kindly carried out by Miss S. Lathwell (National Institute for Medical Research, Mill Hill, London).

Pre-treatment of antigens with various reagents. Antigenic preparations were incubated with concanavalin A (Con A), sodium periodate and pronase and the effect of these reagents on their precipitin reactivities was studied (Hearn \& Mackenzie, 1979).

\section{RESULTS}

Chemical composition. Analysis of the different extracts for protein and carbohydrate content is summarized in Table 1 . The protein to carbohydrate ratio varied widely from species to species, the variation being most noticeable in the WS fraction, where $A$. fumigatus 2140 and $A$. flavus had protein to carbohydrate ratios of 0.7 and 4.8 , respectively. In the TE fraction the protein to carbohydrate ratio increased from 1.0 for A. fumigatus 2109 to 3.4 for $A$. terreus; in the WS/TE fraction the greatest difference in protein to carbohydrate ratio was seen between the $A$. niger $(0 \cdot 6)$ and $A$. terreus $(1 \cdot 6)$ extracts.

The sugar composition, when expressed as a ratio of total neutral sugars to hexosamines, again varied considerably in the WS fraction, from zero hexosamine content in $A$. niger and $A$. nidulans to a neutral sugars to hexosamine ratio of $4 \cdot 2$ for $A$. fumigatus 2140 . All TE preparations contained appreciable quantities of hexosamine, the ratio being lowest for $A$. nidulans (5.9); with the exception of $A$. niger (neutral sugars to hexosamine ratio $5 \cdot 7$ ) the WS/TE fractions contained no measurable amounts of hexosamine.

Recovery of antigenic material. The amount of each fraction recovered was based on its total protein and carbohydrate content. Yields were calculated as a percentage related to the recovery from total dried wall material. The highest recoveries of TE extracts (approx. $3 \%$ ) 
Table 1. Protein and carbohydrate contents of antigenic preparations from Aspergillus species

Ratios of protein to carbohydrate $(P: C)$ and total neutral sugars to hexosamines $(N: H)$ are shown for each antigenic extract. Contents are expressed as $\mathrm{mg}$ recovered from $650 \mathrm{mg}$ dry wt wall. For the WS fraction, samples of 5 to $15 \mathrm{mg}$ dry wt were analysed from the different species.

\begin{tabular}{lccccccc} 
Antigenic & \multicolumn{7}{c}{ Aspergillus species } \\
\cline { 3 - 8 } fraction & Component & $\begin{array}{c}\text { fumigatus } \\
2109\end{array}$ & $\begin{array}{c}\text { fumigatus } \\
2140\end{array}$ & flavus & terreus & niger & nidulans \\
TE & $\mathrm{P}: \mathrm{C}$ & $10 \cdot 1: 9 \cdot 9$ & $13 \cdot 1: 5 \cdot 8$ & $5 \cdot 7: 2 \cdot 5$ & $7 \cdot 8: 2 \cdot 3$ & $4 \cdot 0: 3 \cdot 3$ & $7 \cdot 9: 3 \cdot 4$ \\
& $\mathrm{~N}: \mathrm{H}$ & $7 \cdot 5: 2 \cdot 4$ & $4 \cdot 5: 1 \cdot 3$ & $1 \cdot 5: 1 \cdot 0$ & $1 \cdot 6: 0 \cdot 7$ & $2 \cdot 4: 0 \cdot 9$ & $2 \cdot 9: 0 \cdot 5$ \\
WS/TE & $\mathrm{P}: \mathrm{C}$ & $0 \cdot 9: 0 \cdot 9$ & $0 \cdot 7: 0 \cdot 5$ & $0 \cdot 7: 0 \cdot 5$ & $0 \cdot 8: 0 \cdot 5$ & $0 \cdot 8: 1 \cdot 4$ & $1 \cdot 5: 1 \cdot 1$ \\
& $\mathrm{~N}: \mathrm{H}$ & $0 \cdot 9: 0$ & $0 \cdot 5: 0$ & $0 \cdot 5: 0$ & $0 \cdot 5: 0$ & $1 \cdot 2: 0 \cdot 2$ & $1 \cdot 2: 0$ \\
WS & $\mathrm{P}: \mathrm{C}$ & $5 \cdot 1: 2 \cdot 7$ & $4 \cdot 8: 6 \cdot 8$ & $4 \cdot 8: 1 \cdot 0$ & $5 \cdot 5: 1 \cdot 3$ & $2 \cdot 6: 0 \cdot 6$ & $2 \cdot 4: 0 \cdot 6$ \\
& $\mathrm{~N}: \mathrm{H}$ & $2 \cdot 3: 0 \cdot 4$ & $5 \cdot 5: 1 \cdot 3$ & $0 \cdot 8: 0 \cdot 2$ & $1 \cdot 2: 0 \cdot 1$ & $0 \cdot 6: 0$ & $0 \cdot 7: 0$
\end{tabular}

Table 2. Sugar composition of fractions from Aspergillus species

\begin{tabular}{|c|c|c|c|c|c|c|c|}
\hline \multirow[b]{2}{*}{$\begin{array}{l}\text { Antigenic } \\
\text { fraction }\end{array}$} & \multirow[b]{2}{*}{$\begin{array}{l}\text { Component } \\
\text { sugar }\end{array}$} & \multicolumn{6}{|c|}{ Aspergillus species } \\
\hline & & $\begin{array}{l}\text { fumigatus } \\
2109\end{array}$ & $\begin{array}{l}\text { fumigatus } \\
\quad 2140\end{array}$ & flavus & terreus & niger & nidulans \\
\hline TE & $\begin{array}{l}\text { Mannose } \\
\text { Galactose } \\
\text { Glucose } \\
\text { Glucosamine } \\
\text { Galactosamine }\end{array}$ & $\begin{array}{c}++ \\
++ \\
+ \\
++ \\
+\end{array}$ & $\begin{array}{c}+++ \\
++ \\
+ \\
++ \\
+\end{array}$ & $\begin{array}{l}++ \\
++ \\
+ \\
+ \\
+\end{array}$ & $\begin{array}{c}+++ \\
++ \\
+ \\
++ \\
\pm\end{array}$ & $\begin{array}{c}++ \\
+++ \\
+ \\
++ \\
-\end{array}$ & $\begin{array}{c}++ \\
++ \\
++ \\
+ \\
\pm\end{array}$ \\
\hline WS & $\begin{array}{l}\text { Mannose } \\
\text { Galactose } \\
\text { Glucose } \\
\text { Glucosamine } \\
\text { Galactosamine }\end{array}$ & $\begin{array}{c}+++ \\
++ \\
+ \\
\text { ND } \\
\text { ND }\end{array}$ & $\begin{array}{c}++ \\
++ \\
+ \\
+ \\
+\end{array}$ & $\begin{array}{c}++ \\
+ \\
++ \\
\text { ND } \\
\text { ND }\end{array}$ & $\begin{array}{c}++ \\
++ \\
+++ \\
\text { ND } \\
\text { ND }\end{array}$ & $\begin{array}{c}+++ \\
++ \\
+ \\
\text { ND } \\
\text { ND }\end{array}$ & $\begin{array}{c}+++ \\
+ \\
+ \\
\text { ND } \\
\text { ND }\end{array}$ \\
\hline
\end{tabular}

,,++++++ and \pm indicate high, medium, low and trace concentrations, respectively; - , not detected; ND, not determined.

were found with the two strains of $A$. fumigatus. Yields of the other species were closely allied to each other and were approximately half that of $A$. fumigatus. The highest yields of the WS/TE fractions were obtained from $A$. niger and $A$. nidulans $(0.5 \%$ and $0.4 \%$, respectively); from other species, the recovery was of the order of $0.2 \%$.

Sugar analysis. Neutral sugars present in the TE extracts were identified by g.l.c. With the WS extracts, glucose and galactose were measured using the available kits while mannose was estimated by difference. Amino sugars were determined using an amino acid analyser. The results are summarized in Table 2.

All extracts contained mannose, galactose, glucose and hexosamine. Mannose was the dominant neutral sugar in all extracts except $A$. niger TE, where galactose was the major neutral sugar, and $A$. terreus WS, where glucose predominated. Galactose was present in most extracts in excess of glucose except for $A$. nidulans (both WS and TE) where the galactose to glucose ratio was of the order of $1: 1$ and A. flavus WS where the ratio was $1: 2$. All TE fractions contained both glucosamine and galactosamine, except $A$. niger where the latter was undetectable. Of the WS preparations only $A$. fumigatus 2140 contained appreciable levels of hexosamine, which was found to be made up of approximately equal amounts of glucosamine and galactosamine.

Sensitivity towards homologous antisera. In double diffusion tests with homologous antisera, the TE preparation of each species had very similar reactivity to that of its WS counterpart where, in general, each showed the presence of two precipitating components. The WS/TE preparations showed differences only in the extracts from A. terreus and A. nidulans, 
Table 3. Concentrations of antigenic material used in double diffusion tests

Concentrations are expressed as $\mathrm{mg}$ protein $\mathrm{ml}^{-1}$.

\begin{tabular}{lcccccc} 
& \multicolumn{5}{c}{ Aspergillus species } \\
\cline { 2 - 4 } $\begin{array}{l}\text { Antigenic } \\
\text { fraction }\end{array}$ & $\begin{array}{c}\text { fumigatus } \\
\text { fumigatus }\end{array}$ & flavus & terreus & niger & nidulans \\
WS & 5.10 & 4.75 & 4.80 & 5.50 & 2.60 & 2.40 \\
TE & 2.00 & 2.60 & 1.20 & 1.60 & 1.20 & 1.60 \\
WS/TE & 0.61 & 0.67 & 0.50 & 0.70 & 0.63 & 1.00
\end{tabular}
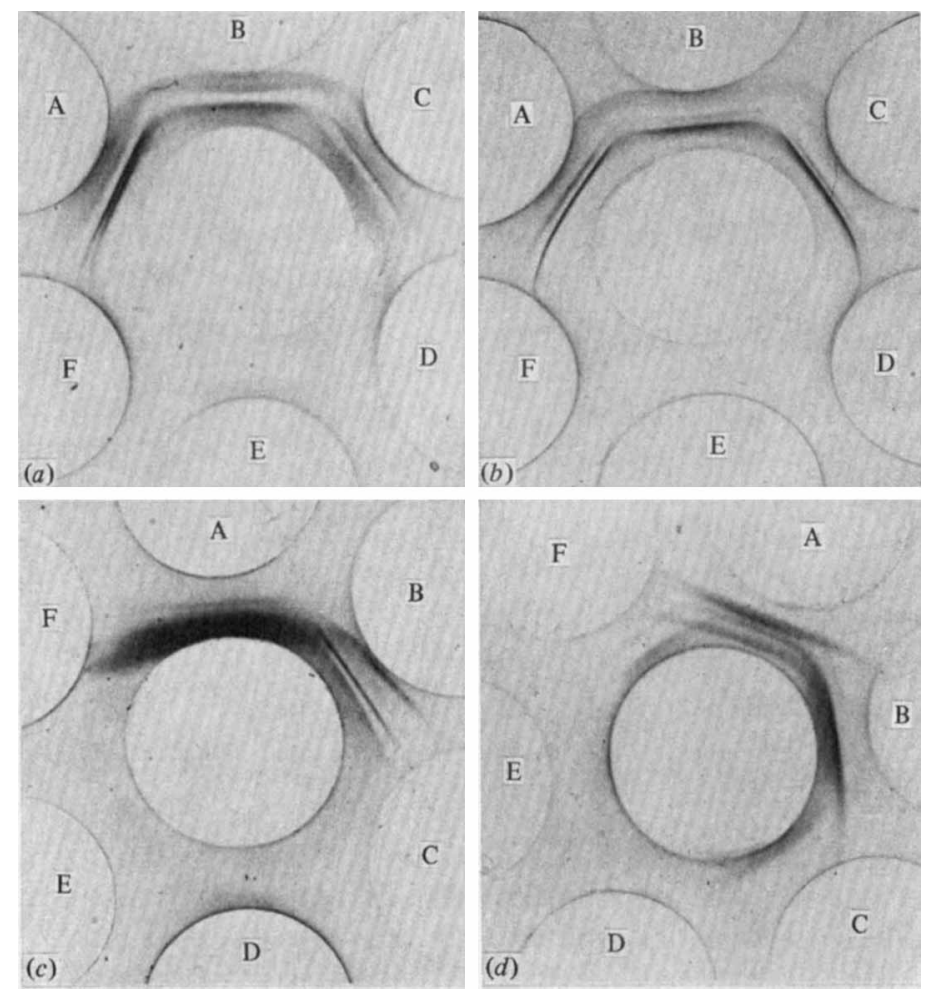

Fig. 1. Sensitivity of antigenic fractions from different Aspergillus species towards human antisera.

(a) Detection of precipitins using a TE fraction from $A$. fumigatus 2109 with aspergilloma and ABPA patients' sera. The centre well contained the antigen. Peripheral wells contained the following sera: A to C, sera from three different aspergilloma patients; D to F, sera from three different ABPA patients. All sera used were undiluted.

(b) Detection of precipitins using a WS/TE fraction from A. fumigatus 2109 with aspergilloma and ABPA patients' sera. The centre well contained the antigen. Peripheral wells contained the same sera as in $(a)$.

(c) Reactivity of the WS antigenic fractions from five Aspergillus species towards human antibodies. The centre well contained an aspergilloma antiserum. Peripheral wells contained the following WS antigens: A, A. fumigatus 2109; B, A. fumigatus 2140; C, A. flavus; D, A.terreus; E, A. niger; F, A. nidulans.

(d) Reactivity of the WS/TE antigenic fractions from five Aspergillus species towards human antibodies. The centre well contained the same aspergilloma antiserum as in $(c)$. The peripheral wells contained the following antigens: A, A. fumigatus $2140 ; \mathrm{B}, A$. fumigatus $2109 ; \mathrm{C}, A$. terreus; D, $A$. nidulans; E, A. niger; F, A. flavus. 


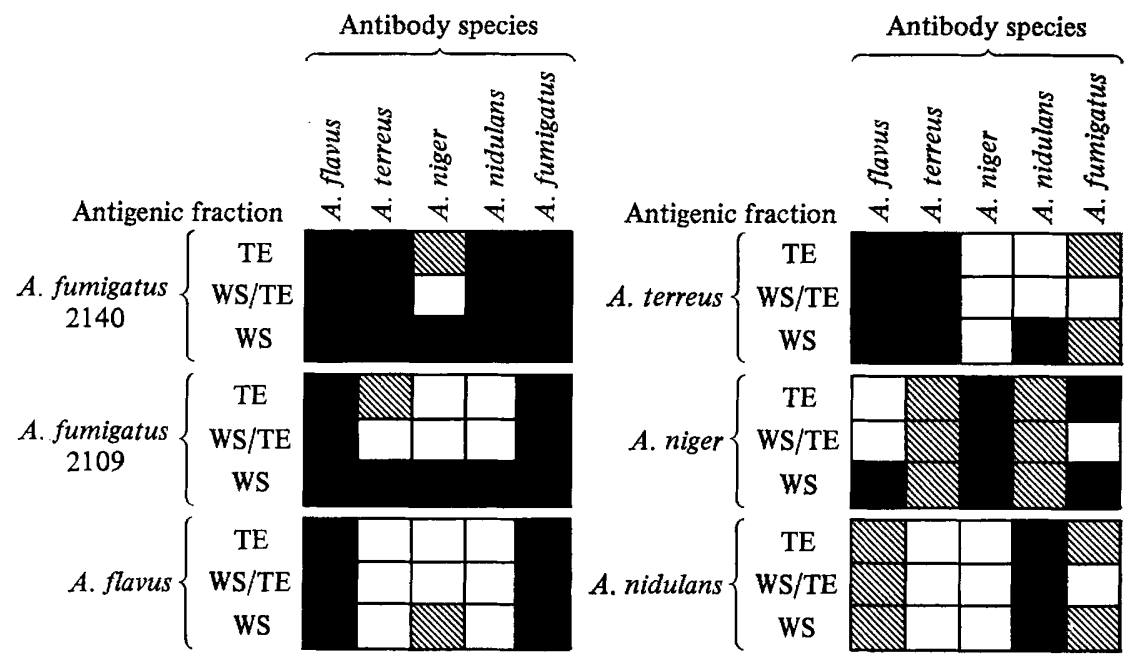

Fig. 2. Reactivity of antigenic fractions towards heterologous antisera. Black areas indicate readily detectable arc(s) of precipitation, shaded areas indicate trace reactivity; white areas indicate no detectable reactivity.

where routinely only one precipitin arc was seen. The levels of antigens used in double diffusion were based on protein content and varied considerably depending on the preparation (Table 3).

Reactivity towards patients' sera. When allowed to react against sera from ABPA patients, weak reactivity was seen with both WS and TE antigen preparations, while reactivity towards a WS/TE fraction was either trace or undetectable (Fig. $1 a, b$ ). On fivefold concentration of individual ABPA sera, and with sera from aspergilloma patients, $4 \pm 1$ precipitin arcs were seen with the WS antigens from the two strains of $A$. fumigatus used. Under similar conditions, the TE and WS/TE fractions gave an average of $3 \pm 1$ precipitin arcs with aspergilloma antisera and an average of $2 \pm 1$ precipitin arcs with concentrated ABPA sera.

Cross-reactivity of patients' antibodies towards $A$. flavus and A. terreus precipitinogens (in six and three cases, respectively) was detectable with all three antigenic fractions (Fig. $1 c, d)$. Two cases of $A$. niger cross-reactivity were detectable with all types of antigen. A single case of an ABPA serum sample which reacted positively with the WS antigen preparation of $A$. nidulans showed no detectable precipitation with the TE and WS/TE extracts.

Reactivity towards heterologous antisera. Each extract was allowed to diffuse against the antisera of the other Aspergillus species under test (Fig. 2). The only major differences were seen with the A.fumigatus 2109 fractions where, in contrast to the WS fraction, the TE and WS/TE preparations showed no cross-reactivity with $A$. niger or $A$. nidulans antisera and the WS/TE fraction failed to cross-react with $A$. terreus antiserum (Fig. $3 a, c$ ). This selectivity was not seen with the other strain of $A$. fumigatus (2140) where the wall antigens reacted with all antisera except that the WS/TE extract showed no detectable cross-reactivity to $A$. niger (Fig. $3 b$ ). The trace reactivity of the WS fraction of $A$. flavus towards $A$. niger antiserum was not seen with the wall antigens while the trace reactivity of the WS and TE fractions of $A$. nidulans towards $A$. fumigatus antiserum was not seen with the WS/TE antigen preparation. Aspergillus terreus cross-reactivity towards $A$. nidulans was lost with the wall fractions and the WS/TE extract showed no reaction with $A$. fumigatus antiserum; with $A$. niger wall antigens a lack of response to $A$. flavus antiserum was noted and the WS/ TE fraction also failed to react with $A$. fumigatus antiserum.

Counterimmunoelectrophoresis. Samples of TE and WS/TE from an A. fumigatus 2109 preparation were tested at different concentrations by counterimmunoelectrophoresis against sheep and human aspergilloma sera and compared with a WS fraction (Fig. $4 a, b$ ). 

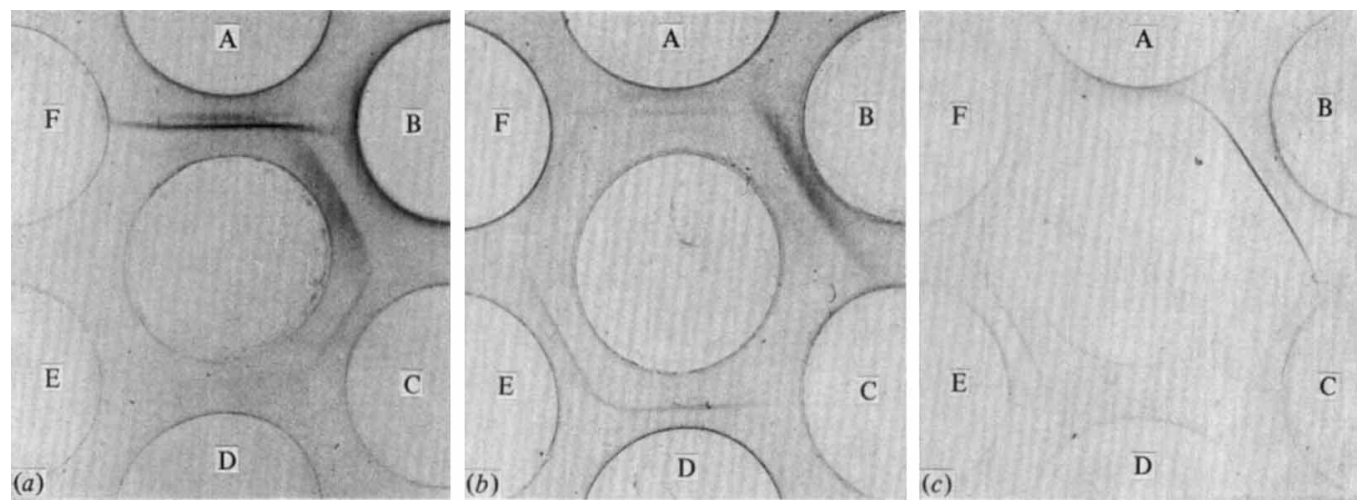

Fig. 3. Reactivity of A. fumigatus antigenic fractions towards antisera from the different Aspergillus species.

(a) Precipitin reactions seen with a TE fraction from A. fumigatus 2109 . The centre well contained the antigen. Peripheral wells contained the following antisera: A, A. fumigatus 2109; B, A. fumigatus 2140; C, A. flavus; D, A. terreus; E, A. niger; F, A. nidulans.

(b) Precipitin reactions seen with a WS/TE fraction from $A$. fumigatus 2140 . The centre well contained the antigen. Peripheral wells contained the following antisera: A, A. fumigatus 2140; B, $A$. fumigatus 2109; C, A. niger; D, A. nidulans; E, A. terreus; F, A. flavus.

(c) Precipitin reactions seen with a WS fraction from $A$. fumigatus 2109 . The centre well contained the antigen. Peripheral wells contained the following antisera: A, A. fumigatus 2109; B, A. fumigatus 2140; C, A. flavus; D, A. terreus; E, A. niger; F, A. nidulans.
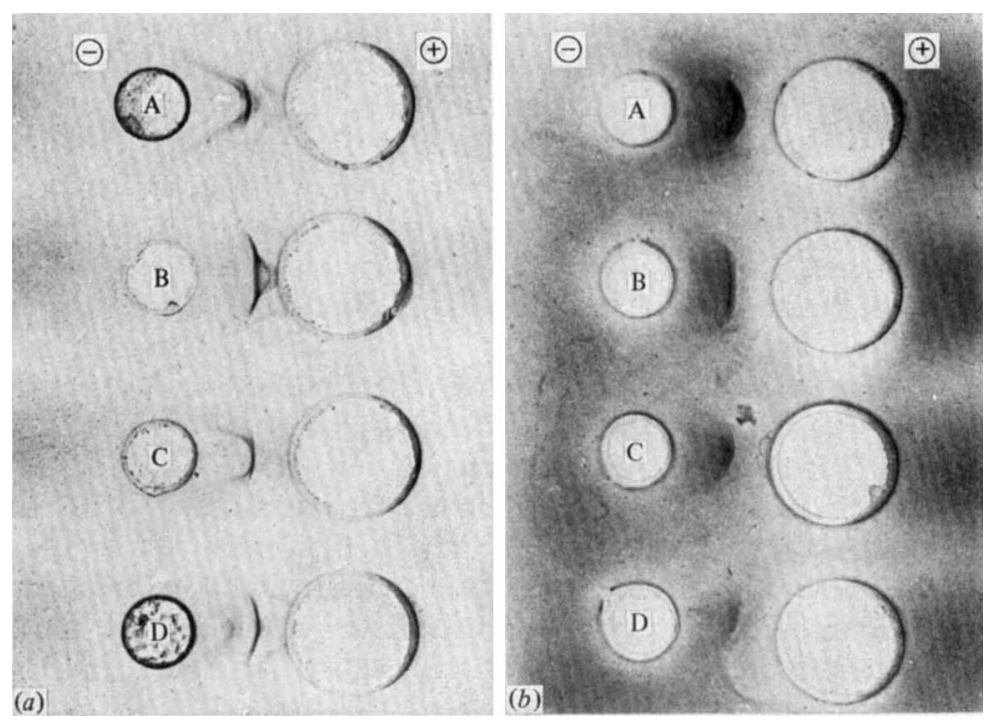

Fig. 4. Behaviour of the three antigenic fractions of A. fumigatus 2109 on counterimmunoelectrophoresis.

(a) A sheep antiserum was used undiluted in the anodal wells. The cathodal wells contained $5 \mu \mathrm{l}$ of the following antigenic extracts (protein contents): A, TE (10 $\mu \mathrm{g})$; B, TE $(5 \mu \mathrm{g}) ; \mathrm{C}$, WS $(60 \mu \mathrm{g})$; $\mathrm{D}, \mathrm{TE}(7.5 \mu \mathrm{g})$.

(b) A human aspergilloma pool was used undiluted in the anodal wells. The cathodal wells contained $5 \mu \mathrm{l}$ of the following antigenic extracts (protein contents): A, WS/TE $(10 \mu \mathrm{g}) ; \mathrm{B}, \mathrm{WS} / \mathrm{TE}$ $(7.5 \mu \mathrm{g}) ; \mathrm{C}, \mathrm{WS} / \mathrm{TE}(5 \mu \mathrm{g}) ; \mathrm{D}$, WS $(60 \mu \mathrm{g})$. 
Table 4. Variation in protein and carbohydrate contents of different batches of A. fumigatus 2109 antigens

Protein and carbohydrate contents of TE and WS/TE preparations are expressed as mg recovered from $10 \mathrm{~g}$ wet wt mycelium; those of the WS extract are expressed as mg recovered from $24 \mathrm{mg}$ dry wt. All results are based on a minimum of six preparations of each extract; standard deviations are given.

$\begin{array}{ccc}\text { Antigenic fraction } & \text { Protein } & \text { Carbohydrate } \\ \text { WS } & 12 \cdot 3 \pm 3 \cdot 6 & 6 \cdot 4 \pm 2 \cdot 6 \\ \text { TE } & 10 \cdot 8 \pm 3 \cdot 2 & 10 \cdot 8 \pm 3 \cdot 0 \\ \text { WS/TE } & 0 \cdot 89 \pm 0 \cdot 2 & 0 \cdot 9 \pm 0 \cdot 25\end{array}$

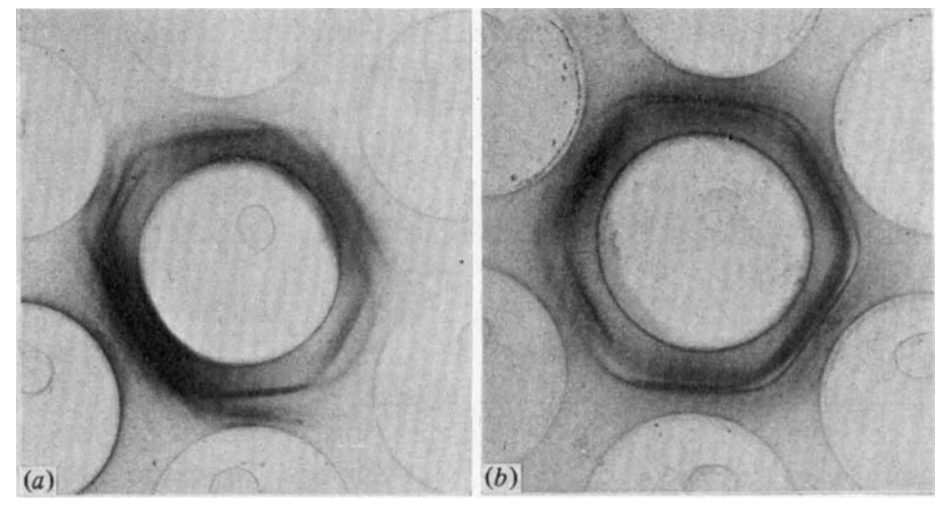

Fig. 5. Comparison of different batches of WS and TE antigens by double diffusion.

(a) Reactivity of WS preparations against a selected aspergilloma serum. The centre well contained the antiserum. The peripheral wells contained samples of six different batches of watersoluble antigens.

(b) Reactivity of TE preparations against the same aspergilloma serum as in (a). The centre well contained the antiserum. The peripheral wells contained samples of six different batches of wall antigens.

The wall antigens were readily detectable at $1.5 \mathrm{mg}$ protein $\mathrm{ml}^{-1}$; the WS extract, on the other hand, was used routinely at $12 \mathrm{mg}$ protein $\mathrm{ml}^{-1}$, as a positive control.

Reproducibility of antigenic preparations of $A$. fumigatus. The WS and wall extracts of A. fumigatus 2109 were examined chemically and immunologically for batch-to-batch variation. The total protein and carbohydrate contents of several preparations were determined (Table 4). Considerable fluctuations were apparent in both. On a comparative basis, the degree of variation between WS, TE and WS/TE extracts was quite similar.

The WS and TE preparations were tested by double diffusion against serum from an aspergilloma patient (Fig. 5a,b). The different batches of TE all showed similar reactivities. While some of the WS preparations had very high reactivities when tested against the same serum, others were much less reactive.

Sensitivity to pronase. Reactivity towards homologous antiserum on double diffusion was lost on pre-incubation of antigen with a proteolytic enzyme. Only with the WS fraction of A. terreus was trace reactivity still retained under these conditions.

Sensitivity to sodium periodate. Periodate oxidation had no effect on antigen activity with the exception of $A$. niger, where antigenic fractions lost one of two precipitin components.

Sensitivity to Con A. Addition of Con A to antigen preparations caused precipitation with subsequent loss of immunological activity. Aspergillus terreus WS fraction was unaffected. 


\section{DISCUSSION}

Using the technique of double diffusion, the immunological reactivities of wall-located antigens from five species of Aspergillus were compared with that of their WS counterparts from whole, macerated mycelium. On a protein basis, an increase in sensitivity towards homologous antiserum was found. The increased sensitivity of the TE fraction relative to the WS fraction was at least twofold while, with the exception of $A$. niger and A. nidulans, an eightfold increase in sensitivity was obtained with the WS/TE fraction. With the one strain of $A$. fumigatus tested by counterimmunoelectrophoresis, the increase in sensitivity of the wall antigens relative to the WS fraction was eight to tenfold, using either homologous antiserum or a human pool, prepared from selected patients' sera.

In general, the fractionation sequence used resulted in some increase in specificity in the antigenic moieties, especially of the WS/TE fractions, as judged by reactivity towards heterologous serum samples. The most noteworthy was one strain of A. fumigatus (2109) where cross-reactivity of the wall antigens to $A$. terreus, $A$. niger and $A$. nidulans antisera was either reduced or was no longer detectable. This was found even when all fractions were tested at comparable protein levels. In this it differed from the other strain of A. fumigatus studied where only cross-reactivity towards $A$. niger was no longer apparent. In counterimmunoelectrophoresis tests carred out in this laboratory, differences in reactivities of the WS fractions of the two strains of $A$. fumigatus when tested against a number of human antisera have also been found (unpublished results).

The small number of patients' sera which reacted positively to more than one species of aspergillus antigen did so to both its WS and wall fractions. Thus, the apparent increase in specificity seen with the WS/TE fractions of $A$. terreus and $A$. niger against experimentally produced heterologous antisera was not found when human specimens were tested. This may be due to quantitative or qualitative differences in antibodies in human compared to experimental samples. At the levels used, the number of precipitin lines seen on double diffusion was lower for wall antigens than for WS preparations tested against both aspergilloma and ABPA sera. However, in no case of proven aspergillosis involvement were the antibodies not detected by the wall fractions.

Chemical analysis showed a sevenfold variation in the protein to carbohydrate ratio from species to species in the WS fraction. Variation was much less marked in the wall fractions, where the difference in the protein to carbohydrate ratio over the species range studied was approximately threefold. On fractionation within a species, enrichment of carbohydrate relative to protein occurred (with the exception of $A$. fumigatus 2140). The TE fractions all contained a high proportion of hexosamine. Removal of Triton resulted in a concomitant loss of hexosamine from solution; the WS/TE fraction contained almost exclusively neutral sugars. Sensitivity to pronase and Con A, but not to periodate oxidation suggests glycoprotein antigens with protein at their active sites.

For the one strain of $A$. fumigatus tested for batch variability, the chemical composition was found to fluctuate widely in all three antigenic fractions. Only the WS/TE fraction showed some chemical homogeneity in its sugar composition. When examined on double diffusion a more uniform precipitin pattern was seen with the TE extract than with the WS extract.

The fractionation scheme proposed and carried out on five species of Aspergillus yielded surface antigens of more uniform chemical composition and increased sensitivity in serological tests when compared with their WS counterparts. Results with human sera showed cross-reactivity between the different species, indicating the presence of shared antigenic determinants in these extracts. Isolation and analysis of common antigens would be of interest in the study of antigen-antibody interactions. At the same time, further fractionation to select immunologically specific entities, linked to a more detailed analysis of their chemical composition, is required to achieve more standardized reagents for serological techniques. It is hoped that these wall-located antigens with their increased sensitivity in in vitro tests can be further purified and characterized to fill this role. 
The authors are indebted to Mrs E. Wilson and Miss M. Angeli for excellent technical assistance. This work was supported by a grant from the Medical Research Council.

\section{REFERENCES}

Biguet, J., Tran van Ky, P., Andrieu, S. \& Fruit, J. (1964). Analyse immunoélectrophorétique d'extraits cellulaires et de milieux de culture d'Aspergillus fumigatus par des immun sérums expérimentaux et des sérums de malades atteints d'aspergillome bronchopulmonaire. Annales de l'Institut Pasteur 107, 72-97.

Clamp, J. R. (1974). Analysis of glycoproteins. Biochemical Society Symposia 40, 3-16.

Coleman, R. M. \& Kaufman, L. (1972). Use of the immunodiffusion test in the serodiagnosis of aspergillosis. Applied Microbiology 23, 301-308.

Dubois, M., Gilles, K. A., Hamilton, J. K., Rebers, P. A. \& SMITH, F. (1956). Colorimetric method for determination of sugars and related substances. Analytical Chemistry 28, 350-356.

HEARN, V. M. \& MACKENZIE, D. W. R. (1979). The preparation and chemical composition of fractions from Aspergillus fumigatus wall and protoplasts possessing antigenic activity. Journal of General Microbiology 112, 35-44.

HeARN, V. M. \& MACKenzie, D. W. R. (1980). Antigenic activity of sub-cellular fractions of Aspergillus fumigatus. Medical Mycology, Pro- ceedings of the XII International Congress of Microbiology, Munich. Edited by H.-J. Preusser. Stuttgart: Gustav Fischer Verlag.

LEE, Y.C. \& MONTGOMERY, K. (1961). Determination of hexosamines. Archives of Biochemistry and Biophysics 93, 292-296.

Longbottom, J. L. \& Pepys, J. (1964). Pulmonary aspergillosis: diagnostic andlimmunological significance of antigens and c-substance in Aspergillus fumigatus. Journal of Pathology and Bacteriology 88, 141-151.

Mackenzie, D. W. R., Proctor, A. G. J. \& Philpot, C. M. (1980). Basic Serodiagnostic Methods for Diseases Caused by Fungi and Actinomycetes, Public Health Laboratory Service Monograph Series no. 12. London: HMSO.

Proctor, A. G. (1976). Mycological methods. In Microbiological Methods, 4th edn., pp. 181-193. London: Butterworths.

STEInBUCH, M. \& AUdRAN, R. (1969). The isolation of IgG from mammalian sera with the aid of caprylic acid. Archives of Biochemistry and Biophysics 134, 279-284. 